VOL. 34 (1986) $395-410$

\title{
LIE GROUP VALUED INTEGRATION IN
}

\section{WELL-ADAPTED TOPOSES}

\author{
ANDERS KOCK
}

In the context of synthetic differential geometry, we prove that group valued 1-forms on the unit interval are exact, provided the group in question is a lie group. This exactness is the basic assumption in a previous paper by the author on differential forms with values in groups.

\section{Introduction.}

We consider the standard well adapted topos models for synthetic differential geometry, and prove the validity here of a fundamental Theorem of differential geometry, namely that, for $G$ a Lie Group, * $\quad G$-valued 1 -forms on $R$ (or on $[0,1]$ ) are exact.

(the classical (well known) version of this Theorem has a less simple formulation, and is stated in the beginning of Chapter 3.) I have expounded the meaning of $*$ in several articles [8], [9], [10].

The main technical tool for proving validity of * in the topos models is a generalization of a Theorem of 0 . Bruno [2] from the 1variable case to the $n$-variable case, and for this generalization, we

Received 6 January 1986. I want to thank Eduardo Dubuc for crucial stimulation and help in the present work, which was carried out while he was visiting Aarhus University on a Danish Natural Science Research Council grant.

Copyright Clearance Centre, Inc. Serial-fee code: 0004-9727/86 $\$ A 2.00+0.00$. 
resort to convenient vector space theory [6], [14], [12].

The well-adapted models we consider are $Z, F$ and $G$ of [4], [15], whose sites of definition have as objects $C^{\infty}$-rings $C^{\infty}\left(I^{n}\right) / I$ with $I$ an arbitrary, respectively $W$-determined, respectively germ determined ideal (terminology of [7]). (The arguments and results we present are independent of which subcanonical Grothendieck topology we consider.) Any of the three toposes will be denoted $E$. The category $M f$ of manifolds is embedded into $E$, in the standard way, $M \in M f$ being represented by the ring $C^{\infty}(M)$. We omit the embedding $i$ from the notation, except that we write $R$ for $i(I R)$.

1. Congruence modulo ideals.

Let $I \subseteq C^{\infty}\left(I R^{P}\right)$ be an ideal, fixed for this section. Let $M$ be a manifold (or any other set structured with a $C^{\infty}$-ring $C^{\infty}(M)$ of functions $M \longrightarrow I R$, in particular, $M$ may be a convenient vector space). We let $I(M)$ denote the equivalence relation on $C^{\infty}\left(I R^{P}, M\right)$ given by $f \equiv g \bmod I(M)$ if and only if for all $\phi \in C^{\infty}(M),(\phi \circ f-\phi \circ g) \in I$ or equivalently, if and only if

$$
C^{\infty}(M) \underset{g^{*}}{\longrightarrow} C^{\infty}\left(I R^{P}\right) \longrightarrow C^{\infty}\left(I R^{P}\right) / I
$$

commutes .

This we call weak congruence mod $I(M)$, or just mod $I$. If $X$ is a convenient vector space, we let $I(X)$ denote the linear subspace of $C^{\infty}(I R, X)$ spanned (purely algebraically) by functions of the form (1.2) $h(t) \cdot k(t) \quad t \in I^{P}$

with $h: I^{P} \longrightarrow I R$ in $I$ and $k: I R^{P} \longrightarrow X$ arbitary smooth . Two maps $\operatorname{IR}^{P} \longrightarrow X$ will be called strongly congruent mod $I(X)$, or just mod $I$, if their difference belongs to $I(X)$.

To compare the two notions where it makes sense (Proposition 1.3 below), we shall use the following unsurprising Lemma from convenient vector space theory. 
LEMMA 1.1. Let $G: X \longrightarrow Y$ be a smooth mop between convenient vector spaces. Then there exists a smooth $H: X \times X \times I R \longrightarrow Y$ such that

$$
G(x+\lambda \cdot y)=G(x)+\lambda \cdot H(x, y, \lambda)
$$

for all $x, y \in X$ and $\lambda \in \mathbb{R}$.

Proof. Consider the function $H$ defined by

$$
H(x, y, \lambda)=\int_{0}^{1} d f_{x+s \lambda y}(y) d s .
$$

It will serve in (1.3), by the standard (Hadamard) calculation. It depends smoothly on $(x, y, \lambda)$; for, $d f_{x}(y)$ depends smoothly on $(x, y)$ (see [14], Satz p.299, or [6], Theorem 6.2), and integration preserves smoothness (see for example [12], Proposition 2.6).

Let $X$ and $Y$ again denote convenient vector spaces; then

PROPOSITION 1.2. Let $f, g: I^{P} \longrightarrow X$ be strongly congruent mod $I$, and let $G: X \longrightarrow Y$ be smooth. Then $G \circ f$ and $G \circ g$ are strongly congment mod $I$.

Proof. By assumption, $g(t)=f(t)+\sum h_{i}(t) k_{i}(t)$ with $h_{i}$ and $k_{i}$ as in (1.2). We may remove one $h_{i}(t) \cdot k_{i}(t)$ summand at a time, so it suffices to consider the case

$$
g(t)=f(t)+h(t) \cdot k(t) .
$$

Let $H$ be as in Lemma 1.1. Then since $h(t) \in I R$, we have

$$
\begin{aligned}
G(g(t)) & =G(f(t)+h(t) \cdot k(t)) \\
& =G(f(t))+h(t) \cdot H(f(t), k(t), h(t)),
\end{aligned}
$$

and the last term is in $I(Y)$ due to the factor $h(t)$.

PROPOSITION 1.3. Let $X$ be a convenient vector space; then strong congruence mod $I$ of mops $I R^{P} \longrightarrow X$ implies weak congmence. For $X$ finite dimensional, the converse holds.

Proof. The first part is immediate from Proposition 1.2 (let $\left.G=\phi \in C^{\infty}(M)\right)$. For the second, let $f$ and $g: I R^{P} \longrightarrow \mathbb{R}^{n}$ be 
weakly congruent mod $I\left(I R^{n}\right)$. For each of the $n$ coordinate projections $\operatorname{proj}_{i}: I R^{n} \longrightarrow I R$, we therefore have

$$
\operatorname{proj}_{i} \circ g-\operatorname{proj}_{i} \circ f \in I \text {. }
$$

Denote this map by $h_{i}: I R^{P} \longrightarrow I R \cdot$ So

$$
g(t)=f(t)+\sum h_{i}(t) \cdot \underline{e}_{i}
$$

where $\underline{e}_{i}$ is the constant function $I R^{P} \longrightarrow I^{n}$ with value $\underline{e}_{i} \epsilon I^{n}$. Since $h_{i} \in I$, this proves strong congruence.

It is clear that strong congruence behaves well with respect to products: for maps $I R^{P} \longrightarrow X_{1} \times \ldots \times X_{n} \quad\left(X_{i}\right.$ convenient), congruence mod $I\left(X_{1} \times \ldots \times X_{n}\right)$ is tested coordinatewise, that is by testing congruence $\bmod I\left(X_{i}\right)(i=1, \ldots, n)$. As a corollary of proposition 1.2, we therefore derive

PROPOSITION 1.4. Let $G: X_{1} \times \ldots \times x_{n} \longrightarrow y$ be smooth, and let $f_{j}, g_{j}$ be mops $I^{P} \longrightarrow X_{j}$. If (strongly)

$$
f_{j} \equiv g_{j} \bmod \quad I\left(X_{j}\right) \quad j=1, \ldots, n
$$

then (strongly)

$$
G \circ\left(f_{1}, \ldots, f_{n}\right) \equiv G \circ\left(g_{1}, \ldots, g_{n}\right) \bmod I(Y) .
$$

If $K$ is a manifold, the ideal $I \subseteq C^{\infty}\left(I R^{P}\right)$ defines an ideal $I^{*}$ in $C^{\infty}\left(I R^{P} \times K\right)$, namely the one spanned by functions $h(t) k(t, x)$ ( $t \in I R^{P}, x \in K$, and $h \in I$ ). Clearly, under the isomorphism (1.4) $\quad C^{\infty}\left(I R^{P}, C^{\infty}(K)\right) \simeq C^{\infty}\left(I R^{P} \times K\right)$, $I\left(C^{\infty}(K)\right) \subseteq C^{\infty}\left(I R^{P}, C^{\infty}(K)\right)$ corresponds to $I^{*}$. Suppose now that we have a smooth map ('operator')

$$
C^{\infty}(K)^{n} \stackrel{G}{\longrightarrow} C^{\infty}(L)
$$

with $L$ a manifold. The composite

(1.6) $C^{\infty}\left(I R^{P} \times K\right)^{n} \simeq C^{\infty}\left(I R^{P}, C^{\infty}(K)\right)^{n} \stackrel{G_{*}}{\longrightarrow} C^{\infty}\left(I R^{P}, C^{\infty}(L)\right) \simeq C^{\infty}\left(I R^{P} \times L\right)$, 
(where $G_{*}$, modulo the identification $C^{\infty}\left(I^{P}, C^{\infty}(K)\right)^{n} \simeq C^{\infty}\left(I R^{P}, C^{\infty}(K)^{n}\right)$, is just "composing with $G$ ") should be considered as "applying $G$ parameterwise in $t \in I R^{P}$ ". Let us denote it by $G / I R^{P}$ (or just $G$ ). Let $f_{i}$ and $g_{i}$ be elements of $C^{\infty}\left(I R^{P} \times K\right)$ for $i=1, \ldots, n$, and let $G$ be as above (1.5). We then have Then

THEOREM 1.5. Let $f_{i}-g_{i} \in I^{*} \subset C^{\infty}\left(I^{P} \times K\right)$, for $i=1, \ldots, n$.

$$
G / I R^{P}\left(f_{1}, \ldots, f_{n}\right)-G / I^{P}\left(g_{1}, \ldots, g_{n}\right) \in I^{*} \subseteq C^{\infty}\left(I R^{P} \times L\right) .
$$

(For $n=1$, this is implicit in Bruno's Theorem 8, [2].)

Proof. The assumption means $\hat{f}_{i} \equiv \hat{g}_{i} \bmod I\left(C^{\infty}(K)\right) \forall i ;$ by Proposition 1.4 ,

$$
G \circ\left(\hat{f}_{1}, \ldots, \hat{f}_{n}\right)=G \circ\left(\hat{g}_{1}, \ldots \hat{g}_{n}\right) \bmod \quad I\left(C^{\infty}(L)\right)
$$

and again this implies congruence mod $I^{*} \subseteq C^{\infty}\left(I R^{P} \times L\right)$ for the exponential adjoints, which are the terms appearing in the Theorem.

Even when the ideal $I \subseteq C^{\infty}\left(I R^{P}\right)$ is $W$-determined, respectively germ-determined, the ideal $I^{*} \subseteq C^{\infty}\left(I^{P} \times K\right)$ may not be, so to get results about the models $F$ and $G$ (see the introduction), we need to take the 'W-radical', respectively 'germ-radial' of $I^{*}$ (terminology of [7]).

It is known (see, for example [15]) that the $W$-radical $\bar{J}$ of an ideal $J \subseteq C^{\infty}\left(I R^{k}\right)$ is its closure in the Frechet space topology on $C^{\infty}\left(I R^{k}\right)$. An unpublished result of Penon says that the germ-radical $\tilde{J}$ similarly is the closure of $J$ in a finer topology on $C^{\infty}\left(I R^{k}\right)$, called the stone-topology in [2], where this topology is described, and a sketch of Penon's result is given.

We shall need the following important result. Let $K$ and $L$ be manifolds, and let $G: C^{\infty}\left(K, I R^{n}\right) \longrightarrow C^{\infty}(L)$ be a smooth operator. Then

THEOREM. (Frölicher [5]). $G$ is continuous with respect to the Frechet topologies. 
THEOREM. (Bruno [2]). $G$ is continuous with respect to the stone topologies.

(Frölicher in fact proves that any (plot-) smooth map between Frechet spaces is continuous. Bruno proves the Theorem quoted only when $K$ and $L$ are coordinate spaces and $n=1$, but his proof carries over immediately.)

Using these Theorems in conjunction with Theorem 1.5 leads to the following result (with notation as in Theorem 1.5):

THEOREM 1.7. Let $\left(f_{i}-g_{i}\right) \in \bar{I}^{*}$ (respectively $\left.\tilde{I}^{*}\right) \subseteq C^{\infty}\left(I^{P} \times K\right)$ for $i=1, \ldots, n$. Then

$G / I^{P}\left(f_{1}, \ldots, f_{n}\right)-G / I R^{P}\left(g_{1}, \ldots, g_{n}\right) \in \bar{I}^{*}$ (respectivezy $\left.\tilde{I}^{*}\right) \subseteq C^{\infty}\left(I R^{P} \times L\right)$

(For $\tilde{I}^{*}$ and $n=1$, this is Bruno's Theorem $8,[2]$.

Proof. For each $i=1, \ldots, n$, let $\left(h_{m}^{i}\right)_{m \in I N}$ be a sequence in $I^{*}$ converging in the relevant topology to $g_{i}-f_{i}$. For each $m$, Theorem 1.5 applies to the $n$-tuple

$$
\left(f_{i}, f_{i}+h_{m}^{i}\right)=1, \ldots, n
$$

to give

$$
G / I^{P}\left(f_{1}, \ldots, f_{n}\right)-G / I^{P}\left(f_{1}+h_{m}^{1}, \ldots, f_{n}+h_{m}^{n}\right) \in I^{*}
$$

As $m \rightarrow \infty$, the right hand term converges to $G / I^{P}\left(g_{1}, \ldots, g_{n}\right)$ by continuity of $G / I^{P}$ (which is a smooth map $C^{\infty}\left(I R^{P} \times K, I R^{n}\right) \longrightarrow$ $C^{\infty}\left(I R^{P} \times L\right)$, hence continuous by the Theorems quoted). So the difference is the one in the Theorem, and is a limit of expressions (1.7) in $I^{*}$, hence in $\bar{I}^{*}$ (respectively $\tilde{I}^{*}$ ).

Consider more generally a smooth operator

$$
C^{\infty}(K)^{n} \longrightarrow C^{\infty}(L, M),
$$

with $K, L$ and $M$ manifolds. Replacing the codomain in (1.6) by

$$
C^{\infty}\left(I R^{P}, C^{\infty}(L, M)\right) \simeq C^{\infty}\left(I R^{P} \times L, M\right)
$$

yields a smooth map $G / \mathbb{R}^{P}$ : 


$$
C^{\infty}\left(I R^{P} \times K\right)^{n} \longrightarrow C^{\infty}\left(I R^{P} \times L, M\right)
$$

With $\sim$ denoting closure for any of the three topologies under consideration (discrete, Frechet, stone), and with $f_{i}, g_{i} \in C^{\infty}\left(I R^{P} \times K\right)$ as before we have

THEOREM 1.7'. If $f_{i}-g_{i} \in \tilde{I}^{*}(i=1, \ldots, n)$, we have

$$
G / I^{P}\left(f_{1}, \ldots, f_{n}\right) \equiv G / I^{P}\left(g_{1}, \ldots g_{n}\right) \bmod \tilde{I}^{*}(M) \text {. }
$$

Proof. The conclusion (1.8) means 'weak congruence' of course. So let $\phi: M \rightarrow I R$ be smooth, and apply Theorem 1.5 (for the discrete case) or Theorem 1.7 to the smooth operator

$$
C^{\infty}(K)^{n} \stackrel{G}{\longrightarrow} C^{\infty}(L, M) \stackrel{\phi *}{\longrightarrow} C^{\infty}(L) \text {. }
$$

\section{The functor $E$.}

Recall that $E$ denotes any of the well adapted toposes $Z, F$ and $G$ mentioned in the introduction. If $M$ is a manifold and $I \subseteq C^{\infty}(M)$, we let $\tilde{I}$ denote its closure in any of the three topologies (discrete, Frechet, Stone), according to whether we read $Z, F$ or $G$, for $E$. Similarly, $\tilde{a}$ denotes coproduct in the sites of definition of either; if $A$ is in the site, $\bar{A} \in E$ denotes the object it represents. Thus $\bar{A} \times \bar{B}=(A \tilde{\otimes} B)^{-}$.

Let $J \subseteq C^{\infty}\left(I R^{P}\right)$ be a closed ideal, $J=\tilde{J}$. For any manifold $K$, we have

$$
C^{\infty}\left(I R^{n}\right) / J \tilde{\otimes} C^{\infty}(K) \simeq C^{\infty}\left(I^{P} \times K\right) / \tilde{J}^{*} ;
$$

this requires a small argument, which we shall not reproduce here (and I thank E. Dubuc for convincing me of its truth in the $G$ case), since we shall only need the result for $K=I^{k}$, where it is evidently true.

If $M$ and $L$ are manifolds, the exponential object $M^{L} \in E$ goes by the global sections functor $\Gamma$ to the set $C^{\infty}(L, M)$ of smooth maps. So a map ('operator')

$$
N^{K} \stackrel{G}{\longrightarrow} M^{L}
$$


in $E$ goes by $\Gamma$ to an operator

$$
C^{\infty}(K, N) \stackrel{\Gamma(G)}{\longrightarrow} C^{\infty}(L, M)
$$

which evidently is (plot-) smooth. A main result in Bruno [2] is that this process can be reversed when $N=I R$ (he also has some inessential restrictions on $K, L, M)$. From Theorem 1.7' we get a generalization of this result to the case $N=I R^{n}$ (obtained independently also by Moerdijk and Reyes):

THEOREM 2.1. Let $K, L$ and $M$ be manifolds. To cony smooth operator $G$ :

$$
C^{\infty}\left(K, \mathbb{R}^{n}\right) \simeq C^{\infty}(K)^{n} \longrightarrow G \longrightarrow C^{\infty}(L, M),
$$

there is a unique map in $E$

$$
\left(R^{n}\right)^{K} \stackrel{E(G)}{\longrightarrow} M^{L}
$$

with $\Gamma(E(G))=G$.

Proof. Let $A=C^{\infty}\left(R^{P}\right) / J$ be an object in the site of definition of $E$ (so $J=\tilde{J}$ ). We must produce a set theoretic map

$$
\left(R^{n}\right)^{K}(A) \stackrel{\varepsilon_{A}}{\longrightarrow} M^{L}(A)
$$

natural in $A$. An element $b$ on the left corresponds, by Yoneda, exponential adjointness, and (2.1), to an $n$-tuple of elements $b_{i} \in C^{\infty}\left(I^{P} \times K\right) / \tilde{J}^{*}$. Let $\beta_{i} \in C^{\infty}\left(I^{P} \times K\right)$ be a representative of $b_{i}$, so that we have a smooth map $\beta=\left(\beta_{1}, \ldots, \beta_{n}\right): I R^{n} \times K \longrightarrow I^{n}$. Consider

$$
\gamma:=G / I R^{P} \text { (B) } \in C^{\infty}\left(I R^{P} \times L, M\right)
$$

We get a $C^{\infty}$-algebra map 'composing with $\gamma^{\prime}$

$$
C^{\infty}(M) \longrightarrow C^{\infty}\left(I R^{P} \times L\right)
$$

If we choose different representatives $\beta_{i}{ }^{\prime}$ for $b_{i}$, (so $\beta_{i}^{\prime}-\beta_{i} \epsilon \tilde{J}^{*}$ ), we get immediately from Theorem 1.7' that $\gamma^{\prime} \equiv \gamma \bmod \tilde{J}^{*}(M)$ (here $\left.\tilde{J}^{*} \subset C^{\infty}\left(I^{P} \times L\right)\right)$; expressing this fact in the style of $(1.1)$, and then taking the corresponding 'dual' diagram in $E$, yields commutativity of 


$$
\bar{A} \times L \longrightarrow{ }_{R}^{P} \times L \stackrel{\gamma \underset{\gamma^{\prime}}{\longrightarrow}}{\longrightarrow},
$$

so that $b$ well-defines a map $\bar{A} \times L \longrightarrow M$, or, equivalenty, an element of $M^{L}(A)$, as desired. Naturality in $A$ is straightforward (at least, the construction was not un-natural). So the map $E(G)$ in $E$ is now declared to be the natural transformation with components ${ }^{\varepsilon} A$.

It is clear that $\Gamma(E(G))$ is just $G$ : put $A=C^{\infty}\left(I R^{0}\right)=I R$, and use $G / I R^{0}=G$ and $(2.2)$.

The uniqueness assertion yet to be proved we separate out as a separate, slightly more general 'faithfulness' assertion, Proposition 2.2 below.

Recall that the unit interval $[0,1]$ is represented in $E$ by the ring $C^{\infty}(I R) / H$, where $H$ is the ideal of functions $I R \rightarrow I R$ that vanish on $[0,1]$.

PROPOSITION 2.2. Let $K$ and $M$ be representable (in particuzar they may be manifolds), and let $L$ be a manifold, or $[0,1]$. Then any two maps $\psi_{1}, \psi_{2}$ :

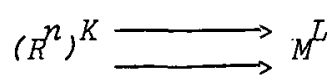

with $\Gamma\left(\psi_{1}\right)=\Gamma\left(\psi_{2}\right)$ are equal.

Proof. Since $M$ is a subobject of some $R^{m}$, we reduce immediately to the case $M=R$, and it suffices to prove that a map $\psi:\left(R^{n}\right)^{K} \longrightarrow \frac{R}{R}$ with $\Gamma(\psi)=0$ is itself 0 . Let $\bar{A}$ be a representable object, and $b: \vec{A} \longrightarrow\left(R^{n}\right)^{K}$. Consideration of the exponential adjoint of $b$, and representability of $K$ (and thus of $\bar{A} \times K$ ) leads to the extension of $b$ to some $c: R^{P} \longrightarrow\left(R^{n}\right)^{K}$, and it suffices to prove $\psi \circ c=0$. Now $\psi \circ c: \frac{P}{R} \longrightarrow \frac{L}{R}$ corresponds to a map $\phi: \frac{P}{R} \times L \longrightarrow R$, such that $\phi(x,-): L \longrightarrow R$ is the zero map for all (global) points $x \in \mathbb{I}^{P}$, by assumption on $\Gamma(\psi)$. In particular, for any (global) point $y \in L$, $\phi(x, y)=0$, so $\phi$ has $\Gamma(\phi)=0$, and since manifolds are fully embedded into $E, \phi$ has to be 0 , for the case when $L$ is a manifold. 
If $L$ is the unit interval, we argue as follows: extend $\phi: R^{P} \times L \longrightarrow R$ into a $\Phi: R^{P} \times R \longrightarrow R$. The smooth function $\Phi: I R^{P} \times I R \longrightarrow I R$ has the property that for each $x \in I^{P}, \Phi(x,-)$ belongs to the ideal $H$, that is $\Phi(x, t)=0 \quad \forall x \in \mathbb{R}^{P}, \forall t \in[0,1]$. But by a deep result of Calderón-Que-Reyes [16], this implies that $\Phi \in H^{*}$, so in particular $\Phi \in \widetilde{H}^{*}$, which is equivalent to saying $\phi=0$.

(The Proposition holds (with the same proof) for any $L$ which is represented by an ideal with line determined extensions in the sense of Bruno [3], which by [3] is a Frechet closed ideal $I$ such that also all $I^{*}$ are Frechet closed)

3. Application to integration in the topos models.

Let $G$ be a Lie group, and $L G$ its tangent space at the neutral element $e \in G$. Consider the pair of operators

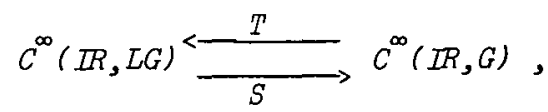

where $T$ is the 'differentiation' operator which to $g: I R \longrightarrow G$ associates $f$ given by

$$
f(t)=\left.\frac{d}{d s}\right|_{s=0} g(t+s) \cdot g(t)^{-1},
$$

and where $S$ to $f: I R \longrightarrow L G$ associates the unique $g$ satisfying (3.1) and $g(0)=e$. (It is a classical result that this $S$ exists and is smooth in parameters. In fact, if $G$ is a matrix group, $g$ is the solution of a linear homogeneous differential (matrix-) equation with variable coefficients $f$.)

The result of the previous section apply to $S$ since $L G \simeq I R^{n}$ (but not to $T$ ). By Theorem 2.1, we get a map $\sigma=E(S)$ with $\Gamma(\sigma)=S$. We also have a $\tau$ in the other direction

$$
L G^{R} \underset{\sigma}{\stackrel{\tau}{\longrightarrow}} G^{R},
$$

namely 'synthetic differentiation', given by the synthetic analogue of (3.1), that is $\tau(g)=f$ with $f$ given by 


$$
f(t)(d)=g(t+d) \cdot g(t)^{-1} \quad \forall d \in D \text {. }
$$

A standard argument, as in [7] III Theorem 3.2, shows that $\Gamma(\tau)=T$. Thus

$$
\Gamma(\tau \circ \sigma)=\Gamma(\tau) \circ \Gamma(\sigma)=T \circ S=i d,
$$

By the 'Faithfulness' Proposition 2.2, $\tau \circ \sigma$ is the identity map on $L G^{R}$

Let us identify the Kernel pair of $\tau$ by a synthetic argument. Suppose $g, h \in G^{R}$ have $\tau(g)=\tau(h)$. Define $g^{-1} \cdot h \in G^{R}$ by

$$
\left(g^{-1} \cdot h\right)(t)=g(t)^{-1} \cdot h(t) \text {. }
$$

Then

$$
\begin{aligned}
\tau\left(g^{-1} \cdot h\right)(t)(d) & =g(t+d)^{-1} \cdot h(t+d) \cdot h(t)^{-1} \cdot g(t) \\
& =g(t+d)^{-1} \cdot \tau(h)(t)(d) \cdot g(t) \\
& =g(t+d)^{-1} \cdot \tau(g)(t)(d) \cdot g(t) \\
& =g(t+d)^{-1} \cdot g(t+d) \cdot g(t)^{-1} \cdot g(t)=e,
\end{aligned}
$$

so $\tau\left(g^{-1} \cdot h\right) \equiv e$ or

$$
\left(g^{-1} \cdot h\right)(t+d)=\left(g^{-1} \cdot h\right)(t) \quad \forall t \in R, d \in D \text {. }
$$

From Proposition 3.1 below it follows that $g^{-1} \cdot h$ is constant, that is there is a unique $c \in G$ so that

$$
h(t)=g(t) \cdot c .
$$

(Conversely, if (3.4) holds, then clearly $\tau(g)=\tau(h)$. )

PROPOSITION 3.1. Let $M$ be a manifold. If $f \in M^{R}$ has $f(t+d)=f(t) \quad \forall t \in R \quad \forall d \in D$, then $f(t)=f(0) \quad \forall t \in R$.

Proof. Since there exists a monic $M \longrightarrow R^{m}$ for some $m$, one quickly reduces the question to the case $M=R$. Let $b: \bar{A} \longrightarrow R^{R}$ be an element at stage $\bar{A}$, and extend it, as in the proof of Proposition 2.2 to an element $c: R^{P} \longrightarrow R^{R}$. Taking exponential adjoints gives an actual map in

$$
\stackrel{P}{R} \times R \longrightarrow R,
$$


and the assumption gives $\frac{\partial \gamma}{\partial t}(x, t) \equiv 0$. The external map $\Gamma(\gamma)$ corresponding to $\gamma$ then has the same property, so $\Gamma(\gamma)=\Gamma(\gamma)(x, 0)$. But $\Gamma$ is faithful on the subcategory $M f \subset E$, so $\gamma(x, t)=\gamma(x, 0) \quad \forall t$ holds internally.

with $G$ a Lie group, and $L G$ its Lie algebra, as above, we derive the following Theorem about $G$-valued integration:

THEOREM 3.2. In $E$ we have

$$
\begin{gathered}
\forall f \in L G^{R} \quad \forall ! g \in G^{R} \text { with } g(0)=e \text { and } \\
g(t+d) \cdot g(t)^{-1}=f(t)(d) \quad \forall t \in R, d \in D .
\end{gathered}
$$

Proof. The (internal) map $E(S)=\sigma$, together with the fact that $\tau \circ \sigma$ is the identity, gives the existence. The uniqueness is immediate from the above identification of the kernel pair of $T$.

The Theorem can be reformulated in terms of differential forms with values in the group $G$, in the sense of [8]:

THEOREM 3.2. In $E$ we hove that any G-valued 1-form on $R$ is exact (with primitive unique modulo right multiplication by a unique constant from G).

Proof. The 1-form $w$ associates with any neighbour pair $(x, y)$ of $R$ an element $w(x, y) \in G$, with $w(x, x)=e \forall x$. Now $(x, y)$ is of the form $(x, x+d)$ for a unique $d \in D$, so

$$
d \longrightarrow w(x, x+d)
$$

defines for each $x \in R$ a tangent vector at $e \in G$. So the information of $w$ is equivalent to that of a curve $R \longrightarrow L G$. The equation (3.5) equivalent to

$$
g(y) \cdot g(x)^{-1}=w(x, y)
$$

for $x$ and $y=x+d$ any neighbour pair of $R$. So $g$ is the primitive, witnessing exactness of $w$. The uniqueness assertion is clearly equivalent to the previous identification of the kernel pair of $\tau$. This proves the Theorem.

We next consider the more important case of Lie group valued integration of functions, defined on the unit interval $[0,1]$. Let $G$ and $L G$ be a Lie group and its Lie algebra, as in Theorem 3.2. 
THEOREM 3.3. In $E$ we hove

$$
\begin{aligned}
& \forall f \in L G^{[0,1]} \quad g ! g \in G^{[0,1]} \text { with } g(0)=e \text { and } \\
& g(t+d) \cdot g(t)^{-1}=f(t)(d) \quad \forall t \in[0,1] \quad \forall d \in D ;
\end{aligned}
$$

equivalently, G-valued 1-forms on $[0,1]$ are exact, with primitive unique modulo right multiplication by a unique constant from $G$.

Proof. The restriction map $L G^{R} \longrightarrow L G^{[0,1]}$ is epic, since $L G \cong R^{n}$ and $[0,1] \longrightarrow R$ is a representable subobject. Equivalently, in a synthetic argument, we may assume that every $[0,1] \longrightarrow L G$ may be extended to $R \longrightarrow L G$, and then the existence assertion follows immediately from the existence assertion in Theorem 3.2. To prove the uniqueness, it suffices to prove that if $g, h \in G^{R}$ have $\left.\tau(g)\right|_{[0,1]}=\left.\tau(h)\right|_{[0,1]}$, with $\tau$ the differentiation process of (3.2), then the function $g \cdot h^{-1}$, defined in (3.3) is constant on $[0,1]$. The same calculation as before yields

$$
\left.\tau\left(g^{-1} \cdot h\right)\right|_{[0,1]} \equiv e .
$$

so the result will follow from the analogue of Proposition 3.1:

PROPOSITION 3.4. Let $M$ be a manifold. If $f \in M^{R}$ has $f(t+d)=f(t) \quad \forall t \in[0,1] \forall d \in D$, then $f(t)=f(0) \forall t \in[0,1]$.

Proof. As in the proof of Proposition 3.1, it suffices to consider the case $M=R$, and again, to consider a generalized element $f$ of $R^{R}$ at stage $\bar{A}=R^{P}, f: R^{P} \longrightarrow R^{R}$. The exponential adjoint $\gamma: R^{P} \times R \longrightarrow R$ satisfies by assumption

$$
\frac{\partial \gamma}{\partial t}(x, t)=0 \quad \forall x, \forall t \in[0,1],
$$

so for $\mathrm{r}(\gamma): \mathbb{R}^{P} \times \mathbb{I R} \longrightarrow \mathbb{R}$, we have, for all $x \in \mathbb{R}^{P}$,

$$
\Gamma(\gamma)(x, t)-\Gamma(\gamma)(x, 0)=0 \quad \forall t \in[0,1] .
$$

This means that the composite of $f$ with the restriction map

$$
R_{R} \longrightarrow R^{R} \longrightarrow R^{[0,1]}
$$


has the property that $\Gamma$ takes it to the zero map. By Proposition 2.2 (which here is really the Calderón-Quel-Reyes result!), the map (3.6) itself is the zero map, and the validity of $f(t)=f(0) \forall t \in[0,1]$ follows. We remark that specializing Theorem 3.3 to the case $G=(R,+)$ gives the validity of the usual "integration axiom" of [13]. The validity of this for the topos $F$ was first proved in Belair [1], and for the topos $G$ was known to Reyes, Dubuc and Penon. For the "Cahiers topos" $C$ (terminology of [7]), the arguments of the present article require some modifications, since $[0,1]$ in no longer representable; but the category of manifolds with boundary is neverthless fully embedded in $C$ which should make the modification of the arguments easy. Anyway, for $C$, we gave an independent proof of $R$-valued integration in [13], and this argument may be extended to give $G$-valued integration for $\mathcal{C}$, as pointed out in $[8]$.

Let us also remark that the 'lifting" of smooth operators

$$
C^{\infty}(K, N) \longrightarrow C^{\infty}(L, M)
$$

to the Cahiers topos, in the case where $N$ and $M$ are vector spaces $I R^{n}$ (or convenient vector spaces) alternatively may be seen as an immediate consequence of the full embedding of convenient vector spaces into $\mathcal{C}$, $[11]$.

We finish by proving the validity of a simple comprehensive form of the Frobenius Theorem. Recall [8] that a G-valued 1-form $w$ on a manifold $M$ is a law which to each neighbour pair $x, y$ of $M$ associates an element $w(x, y) \in G$ with $w(x, x)=e$, and that $w$ is called closed if

$$
w(y, z) \cdot w(x, y)=w(x, z)
$$

whenever $x, y$ and $z$ are mutual neighbours. If $f: M \longrightarrow G$ is a function, $d f$ is the 1 -form on $M$ given by

$$
d f(x, y)=f(y) \cdot f(x)^{-1} \text {, }
$$

and $d f$ is clearly closed; 1-forms of form $d f$ are called exact, and $f$ is called a primitive of $f$.

Let $E$ be any of the well adapted topos models mentioned in the introduction. 
THEOREM 3.5. Let $G$ be a lie group, and $M$ a connected, simply connected manifold*. Then any closed G-valued 1 -form on $M$ is exact (with primitive unique modulo a unique constant $\epsilon G$ ).

Proof. By Theorem 3.3, the group $G$ "admits integration" in the sense of [8] (6.1). The result then follows from loc.cit Theorem 7.2.

\section{References}

[1] Luc Belair, Caloul infinitesimal en gometrie differentielze synthetique, (M.Sc. Thesis, Univ. de Montréal, Aug. 1981).

[2] Oscar P. Bruno, "Vector fields on $\mathrm{R}^{\mathrm{R}}$ in well adapted models of synthetic differential geometry", J. Pure Appl. Algebra 41 (1986).

[3] Oscar P. Bruno, "A property of ideals of differentiable functions", Bull. Austral. Math. Soc. 33 (1986), 293-305.

[4] E.J. Dubuc, "C schemes", Amer. J. Math. 103 (1981), 683-690.

[5] A. Frölicher, "Applications lisses entre espaces et varietés de Frechet", C. R. Acad. Sci. Paris Ser. I Math. 293 (1981), 125-127.

[6] A. Frölicher, B. Gisin and A. Kriegl, "General differentiation theory" in Category Theoretic Methods in Geometry, (Aarhus, Various Publ. Series 35 1983).

[1] A. Kock, Synthetic Differential Geometry, (London Math. Soc. Publ. Series 51 Cambridge Univ. Press 1981).

[8] A. Kock, "Differential forms with values in groups", Bulz. Austral. Math. Soc. 25 (1982), 357-386.

[9] A. Kock, "A combinatorial theory of connections", in Mathematical Applications of Category Theory, J. Gray (Ed.) (Contemp. Math. vol. 30 1984), 132-144.

[10] A. Kock, "Combinatorial notations relating to principal fibre bundles", J. Pure Appl. Algebra 39 (1986), 141-151.

[11] A. Kock, "Convenient vector spaces embed into the Cahiers topos", Cahiers Topologie Geom. Différentielle 27 (1986).

[12] A. Kock, "Calculus of smooth functions between convenient vector spaces", Aarhus Preprint Series 18 (1984/85).

* These connectedness conditions should hold internally in $E$. When this follows from the external condition has still to be investigated. 
[13] A. Kock and G.E. Reyes, "Models for synthetic integration theory", Math. Scand. 48 (1981), 145-152.

[14] A. Kriegl, "Eine kartesisch abegeschlossene Kategorie glatter Abbildungen zwischen beliebigen lokalkonvexen vektorräumen", Monatsh. Math. 95 (1983), 287-309.

[15] I. Moerdijk and G.E. Reyes, $C^{\infty}$-rings, (to appear).

[16] G.E. Reyes and N. Van Que, "Smooth functors and synthetic calculus", (The L.E.J. Browwer Centenary Symposium, A.A. Troelstra and D. van Dalen (Eds.) North Holland, 1982), 377-398.

Matematisk Institut

Aarhus University

Ny Munkegade

8000 AARHUS C. DANMARK

Added in Proof: The arguments for Propositions 3.1 and 3.4 are not quite complete. 